Honam Mathematical J. 36 (2014), No. 4, pp. 787-794

http://dx.doi.org/10.5831/HMJ.2014.36.4.787

\title{
THREE CONVEX HULL THEOREMS ON TRIANGLES AND CIRCLES
}

\author{
Bahman Kalantari AND Jong Youll PARK*
}

\begin{abstract}
We prove three convex hull theorems on triangles and circles. Given a triangle $\triangle$ and a point $p$, let $\triangle^{\prime}$ be the triangle each of whose vertices is the intersection of the orthogonal line from $p$ to an extended edge of $\triangle$. Let $\triangle^{\prime \prime}$ be the triangle whose vertices are the centers of three circles, each passing through $p$ and two other vertices of $\triangle$. The first theorem characterizes when $p \in \triangle$ via a distance duality. The triangle algorithm in [1] utilizes a general version of this theorem to solve the convex hull membership problem in any dimension. The second theorem proves $p \in \triangle$ if and only if $p \in \triangle^{\prime}$. These are used to prove the third: Suppose $p$ be does not lie on any extended edge of $\triangle$. Then $p \in \triangle$ if and only if $p \in \triangle^{\prime \prime}$.
\end{abstract}

\section{Introduction}

We begin with three figures in order to describe three convex hull properties of a general triangle to be proved here.

In Figure 1 we have a point $p$ inside a triangle $\triangle A B C$. We have drawn three circles each centered at a vertex passing through $p$. Note that the three corresponding discs intersect only at $p$. Will this property always be valid? What happens if $p$ is outside of $\triangle A B C$ ?

We mention that a well-known theorem, "Johnson's three circle theorem" is a result on three circles of equal radius $r$ passing through a common point: The circle that passes through the three centers also has radius $r$. Johnson's Theorem is visually evident. However, a natural question is to characterize the cases when the circles do pass through a common point. This characterization will follow here as a special case.

Received September 15, 2014. Accepted September 30, 2014.

2010 Mathematics Subject Classification. 51: Geometry, 52: Convex geometry and discrete geometry.

Key words and phrases.

${ }^{*}$ Corresponding author 


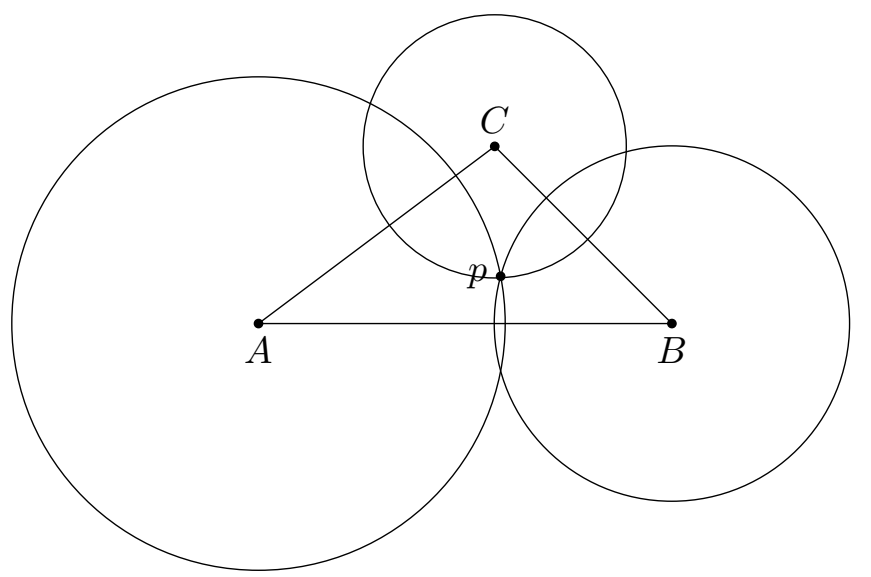

Figure 1. Three circles centered at the vertices of $\triangle A B C$ passing through $p$.

In Figure 2 we have a point $p$ inside a triangle $\triangle A B C$. We have drawn three circles, each passing through $p$ and two other vertices of $\triangle A B C$. As in the previous case the three discs intersect only at $p$. Note that $p$ lies in the triangle whose vertices are the centers of these circles. Will this property always be valid? What happens if $p$ is outside of $\triangle A B C$ ?

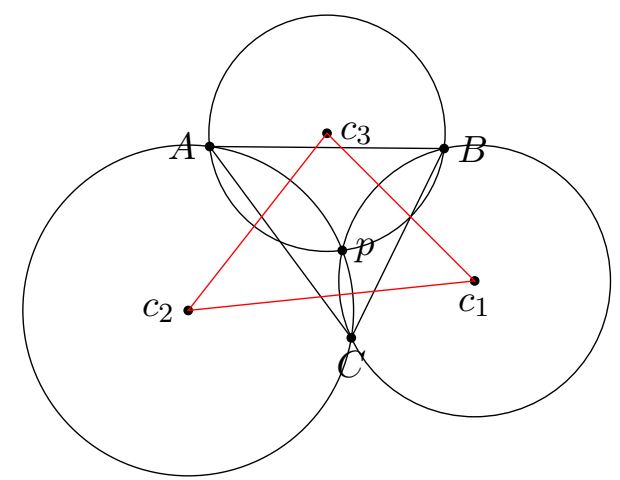

FiguRE 2. Three circles, each passing through $p$ and two other vertices of $\triangle A B C$. 
In Figure 3 also we have a point $p$ inside a triangle $\triangle A B C$. We have drawn three perpendicular lines from $p$ to each of the edges. Denoting the intersection points by $b_{1}, b_{2}, b_{3}$, note that $p$ is inside $\triangle b_{1} b_{2} b_{3}$. Will this property always be valid? What happens if $p$ is outside of $\triangle A B C$ ?

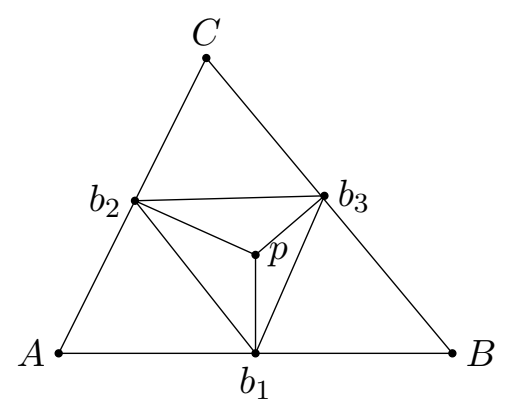

Figure 3 . The triangle of the three intersection of orthogonal lines from $p$ to the edges of $\triangle A B C$.

These three figures should make it clear what we intend to prove. In the next section we will state precise statements and prove three convex hull theorems.

\section{Three Convex Hull Theorems on a Triangle}

The first theorem to be proved, Theorem 1, is proved in much more generality in [1] and is explored in an algorithm called triangle algorithm, solving a range of problems, including the convex hull membership problem in arbitrary dimensions, linear programming, and a linear system of equations. For some related articles that also make comparisons with some algorithms for solving the convex hull problem, linear programming and iterative methods for solving a linear system, see [1]-[6]. For the sake of completeness here we prove the theorem for the special case of a triangle considered in this article.

Theorem 1. (distance duality) Given a triangle $\triangle A B C$, a point $p$ in the plane of the triangle, draw three circles centered at the vertices, passing through $p$. Let $D_{1}, D_{2}, D_{3}$ be the corresponding discs. Then $p \in \triangle A B C$ if and only if $D_{1} \cap D_{2} \cap D_{3}=\{p\}$, i.e. the three discs intersect only at $p$. 
Proof. Suppose $p \in \triangle A B C$ but $D_{1} \cap D_{2} \cap D_{3}$ contains a point $p^{\prime} \neq p$. Consider the orthogonal bisecting line of the line segment $p p^{\prime}$. Since both $p$ and $p^{\prime}$ lie inside the triangle this line when extended must intersect the edges of the triangle, see left figure in Figure 4 . This implies there is a vertex that is closer to $p$ than to $p^{\prime}$ ( $B$ or $C$ in the figure). But this implies that the disc centered at this vertex will exclude $p^{\prime}$, a contradiction.
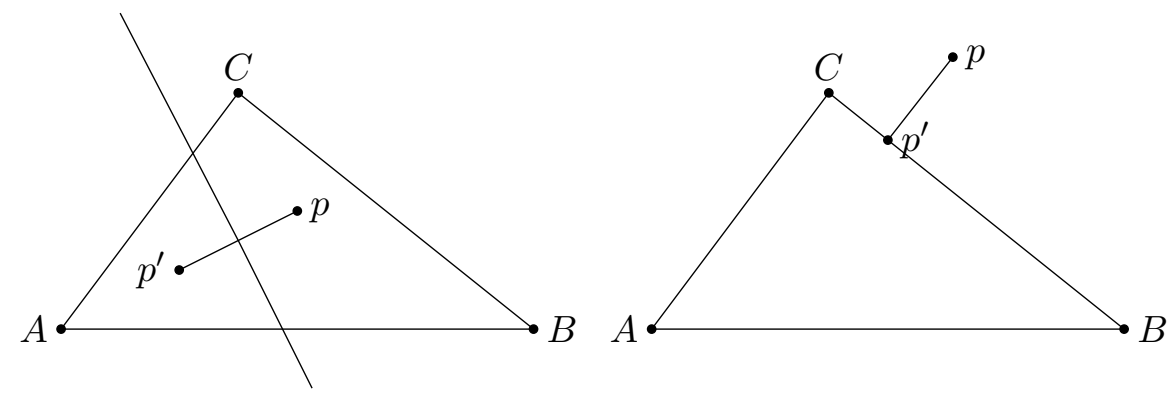

FiguRE 4. The orthogonal bisecting line of $p p^{\prime}$ does not separate $p$ from $\triangle A B C$ (left). The orthogonal bisecting line of $p p^{\prime}$ (not drawn) separates $p$ from $\triangle A B C$ (right).

Conversely, suppose $p \notin \triangle A B C$. Let $p^{\prime} \in \triangle A B C$ be the closest point of the triangle to $p$. Such a point must exist. The orthogonal bisector of $p p^{\prime}$ defines a half-space that contains $p^{\prime}$, and $\triangle A B C$. Thus each vertex is closer to $p^{\prime}$ than to $p$ (see right figure in Figure 4). Hence $p^{\prime} \in D_{1} \cap D_{2} \cap D_{3}$.

Theorem 2. Given a triangle $\triangle A B C$, a point $p$ in the plane of the triangle, draw a perpendicular line from $p$ to each of the extended edges, resulting in three intersection points $b_{1}, b_{2}, b_{3}$. Then $p \in \triangle A B C$ if and only if $p \in \triangle b_{1} b_{2} b_{3}$ (see Figure 3 for an example).

Proof. Assume $p \in \triangle A B C$ but not a boundary point since otherwise the theorem is trivially true. Suppose each $b_{i}$ lies in $\triangle A B C$, see Figure 3. Without loss of generality we assume $A=(0,0)$ in the Euclidean plane, $B=(1,0)$ and $C$ a point with positive $y$-coordinate. Let $p=\left(p_{1}, p_{2}\right)$. Then $0<p_{1}<1$. Note that $b_{1}=\left(p_{1}, 0\right)$ is one of the intersection points. Since $p$ is inside $\triangle A B C$, it is easy to argue the $x$-coordinate of $b_{2}$ and $b_{3}$ must lie to the left and right of $p_{1}$. Since $b_{2}, b_{3}$ are in $\triangle A B C$, a convex set, the line segment $b_{2} b_{3}$ must lie in $\triangle A B C$. Hence there is 
point of the form $\left(p_{1}, y\right) \in \triangle A B C$ where $y>p_{1}$. But this point can be written as a convex combination of $b_{2}$ and $b_{3}$. We can write $p$ as a convex combination of $b_{1}$ and $\left(p_{1}, y\right)$. Hence $p$ can be written as a convex combination of $b_{1}, b_{2}, b_{3}$. But a point lies in a triangle if and only if it can be written as a convex combination of its vertices. Hence $p \in \triangle b_{1} b_{2} b_{3}$.

Next suppose that $p \in \triangle A B C$ but some $b_{i}$ is not in $\triangle A B C$. Without loss of generality we may assume the edge $A B$ is the line segment $[0,1]$ and $p=\left(p_{1}, p_{2}\right)$ with $p_{1}<0$, see Figure 5 . One of the intersections, say $b_{1}$, is $\left(p_{1}, 0\right)$ and it is not in $\triangle A B C$. Since $p \in \triangle A B C$ we can easily argue that both $b_{2}, b_{3}$ must lie in $\triangle A B C$. Thus the line segment $b_{2} b_{3}$ lies in $\triangle A B C$ and contains a point of the form $\left(p_{1}, y\right)$ with $y_{1}>p_{1}$. Then $p$ can be written as a convex combination of $b_{1}$ and $\left(p_{1}, y\right)$, hence a convex combination of $b_{i}^{\prime}$ s. Thus $p \in \triangle b_{1} b_{2} b_{3}$.

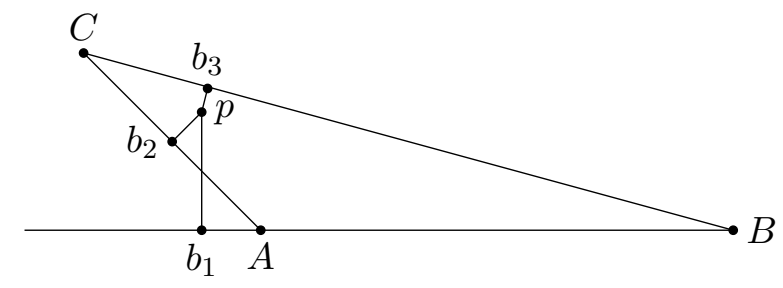

Figure 5. A case where one $b_{i}$ is not in $\triangle A B C$, however $p \in \triangle b_{1} b_{2} b_{3}$.

Conversely, suppose $p \in \triangle b_{1} b_{2} b_{3}$. We claim $p \in \triangle A B C$. Since $p$ is contained in $\triangle b_{1} b_{2} b_{3}$, it is also contained in the three inequalities (half-spaces) defined by the lines orthogonal to $p b_{i}$ at $b_{i}, i=1,2,3$. Let $P$ be the intersection of the three half-spaces. The extreme points of $P$ are precisely $A, B, C$, and lie in $P$. By convexity of $P$, the convex hull of $A, B, C$, namely $\triangle A B C$ coincides with $P$ and hence contains $\triangle b_{1} b_{2} b_{3}$.

Theorem 3. Given a triangle $\triangle A B C$, let $p$ be a point in the plane of the triangle, not lying on any extended edge. For each pair of vertices of $\triangle A B C$, draw a circle that passes through them and also $p$. Let $D_{i}$, $i=1,2,3$ denote the corresponding discs, with centers $c_{i}$. The following conditions are equivalent

(i) $p \in \triangle A B C$; 
(ii) $p \in \triangle c_{1} c_{2} c_{3}$;

(iii) $D_{1} \cap D_{2} \cap D_{3}=\{p\}$.

Proof. Assume (i) holds. By the assumption of the theorem $p$ does not lie on an edge, $p$ is an interior point of $\triangle A B C$ (see Figure 2 or Figure 6 for examples). Consider the triangle whose vertices are the midpoints of the line segments $A p, B p, C p$. Let these midpoints be $A^{\prime}$, $B^{\prime}, C^{\prime}$, respectively. Clearly, $p$ is also in $\triangle A^{\prime} B^{\prime} C^{\prime}$, see Figure 6 . Note that $A^{\prime}, B^{\prime}, C^{\prime}$ are precisely the intersections of orthogonal lines from $p$ to the extended edges of $\triangle c_{1} c_{2} c_{3}$. This follows by the simple fact that the orthogonal bisecting line of each of the line segments $A p, B p$, $C p$ must contain two of the centers of the circles. Thus by Theorem 2, $p \in \triangle c_{1} c_{2} c_{3}$. Thus (i) implies (ii). By Theorem 1, $D_{1} \cap D_{2} \cap D_{3}=\{p\}$. Thus (ii) implies (iii).

Next we prove (iii) implies (i). Suppose $D_{1} \cap D_{2} \cap D_{3}=\{p\}$. By Theorem 1, $p \in \triangle c_{1} c_{2} c_{3}$. By Theorem 2, $p$ is in the triangle whose vertices are the intersection points with respect to orthogonal lines to extended edges of $\triangle c_{1} c_{2} c_{3}$. Let these be $b_{1}, b_{2}, b_{3}$. Extending the line segments $p b_{1}, p b_{2}, p b_{3}$, from $p$ toward $b_{i}$, while doubling the corresponding length, we obtain the vertices $A, B$ and $C$. Hence $p \in \triangle b_{1} b_{2} b_{3}$ implies $p \in \triangle A B C$.

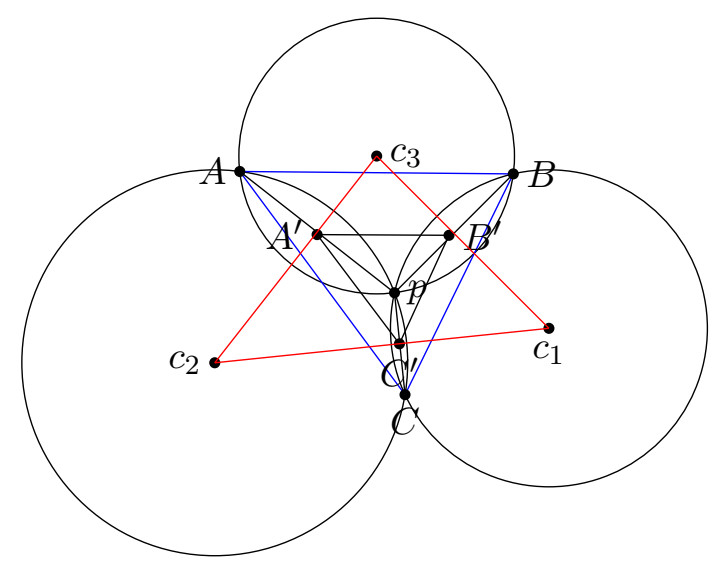

FiguRE 6. Three circles, each passing through $p$ and two other vertices of $\triangle A B C$.

To see what happens when $p$ is not in $\triangle A B C$, we give an example in Figure 7. 


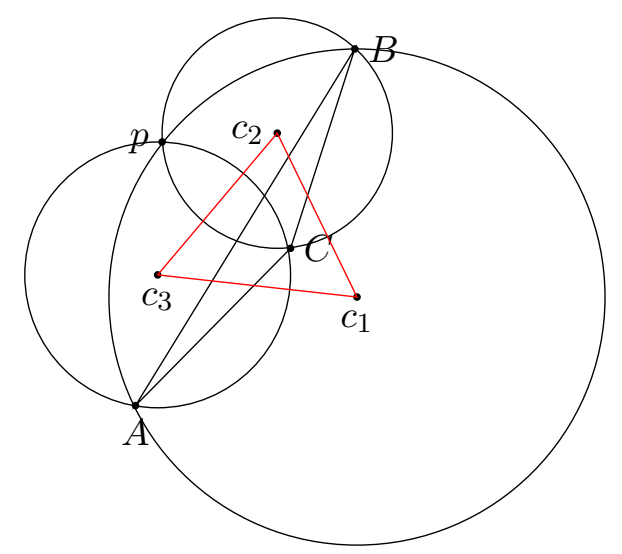

FigURE 7. Three circles, each passing through $p$ and two other vertices of $\triangle$.

\section{Conclusions}

In this article we have proved three convex hull theorems on triangles and circles, revealing interesting properties on these fundamental geometric figures. The results could give rise to generalizations to convex polygons and more generally convex polytopes in arbitrary dimension. Specifically, a generalization of Theorem 1 is given in [1]. We will give generalizations of Theorem 2 and 3 elsewhere. For instance Theorem 2 is generalizable to a simplex of $n+1$ points in the $n$ dimensional Euclidean space.

\section{References}

[1] B. Kalantari, A characterization theorem and an algorithm for a convex hull problem, to appear in Annals of Operations Research, available online August, 2014. arxiv.org/pdf/1204.1873v2.pdf, and http://arxivweb3.library.cornell.edu/pdf/1204.1873v4.pdf, 2012. To appear in Annals of Operations Research, 2014.

[2] B. Kalantari, Finding a lost treasure in convex hull of points from known distances, In the Proceedings of the 24th Canadian Conference on Computational Geometry (2012), 271-276.

[3] B. Kalantari, Solving linear system of equations via a convex hull algorithm, arxiv.org/pdf/1210.7858v1.pdf, 2012. 
[4] B. Kalantari and M. Saks, On the Triangle Algorithm for the Convex Hull Membership, 2-page Extended Abstract, 23nd Annual Fall Workshop on Computational Geometry, City College of New York, 2013.

[5] M. Li and B. Kalantari, Experimental Study of the Convex Hull Decision Problem via a New Geometric Algorithm, 2-page Extended Abstract, 23nd Annual Fall Workshop on Computational Geometry, City College of New York, 2013.

[6] T. Gibson and B. Kalantari, Experiments with the Triangle Algorithm for Linear Systems, 2-page Extended Abstract, 23nd Annual Fall Workshop on Computational Geometry, City College of New York, 2013.

[7] R. Johnson, A circle theorem, Amer. Math. Monthly 23 (1916), 161-162.

\section{Bahman Kalantari}

Department of Computer Science, Rutgers University,

New Brunswick, New Jersey, USA.

E-mail: kalantari@cs.rutgers.edu

Jong Youll Park

Department of Mathematics, Chonnam National University,

Gwangju, South Korea.

E-mail: parkjy@chonnam.ac.kr 